\section{La medalla}

\section{Ismael Cosío}

Villegas-Donato G.

Alarcón:

\section{El mayor}

reconocimiento otorgado por la SMNyCT

Durante el pasado 76 Congreso de las Américas de Neumología y Cirugía de Tórax de la SMNyCT celebrado del 17 al 21 de abril de 2017, en la hermosa ciudad de Puebla de los Ángeles, la Mesa Directiva 2015-2017 que tuve el honor de presidir distinguió a tres de sus más destacados miembros, todos ellos expresidentes de nuestra Sociedad con la Medalla que lleva el nombre de los fundadores de la SMNyCT, los doctores Ismael Cosío Villegas y Donato G. Alarcón.

Está distinción fue entregada a los doctores Octavio Rivero Serrano (Presidente 1965-1966), Raúl Cicero Sabido (1966-1967) y Jaime Villalba Caloca (1989-1991). En una emotiva presentación realizada durante la ceremonia inaugural de este magno evento, donde se leyó una semblanza biográfica de cada uno de los homenajeados por los doctores Francisco Navarro Reynoso (Dr. Rivero), Alfredo Pérez Romo (Dr. Cicero) y Jorge Salas Hernández (Dr. Villalba).

Nuestros galardonados recibieron la presea de manos de los integrantes del presídium que estuvo integrado por el Presidente de la SMNyCT, la Dra. Mayra Mejía Ávila (vicepresidente), el Dr. Andrés Palomar Lever, expresidente de la Sociedad y actual Presidente de la Asociación Latinoamericana del Tórax (ALAT), Dr. Carlos Robalo de Portugal y actual Secretario General de la Sociedad Europea Respiratoria (ERS) y la Dra. Maricela Caleco Torres, Gobernadora del Capítulo de la Sociedad del Estado de Puebla.

En la pasada Asamblea General de Socios y a propuesta de mi equipo de trabajo, se aprobó unánimemente que la Medalla Ismael Cosío Villegas-Donato G. Alarcón se instituya como el mayor reconocimiento que otorgue la SMNyCT. Por acuerdo general, este reconocimiento se entregará una vez al año a distinguidas personalidades nacionales o extranjeras que cuenten con una gran trayectoria profesional y que hayan hecho una gran aportación a la medicina respiratoria y a la SMNyCT. Los candidatos deberán ser propuestos y aprobados por el Comité Ejecutivo de la Sociedad, pero también podrán ser propuestos por distinguidos miembros.
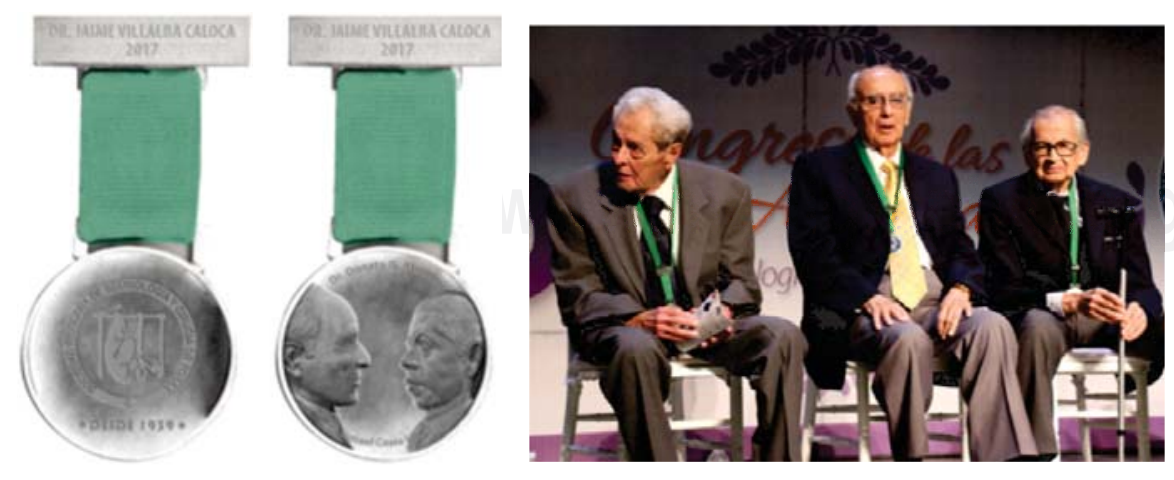

Estamos seguros que esta presea ha sido y será un reconocimiento a la altura y prestigio de nuestra Sociedad y de sus galardonados.

Muchas felicidades por este merecido reconocimiento al Dr. Octavio Rivero Serrano, al Dr. Raúl Cicero Sabido y al Dr. Jaime Villalba Caloca quienes, sin duda, dejan un gran legado a la Medicina Respiratoria y a la Medicina en general de nuestro país.

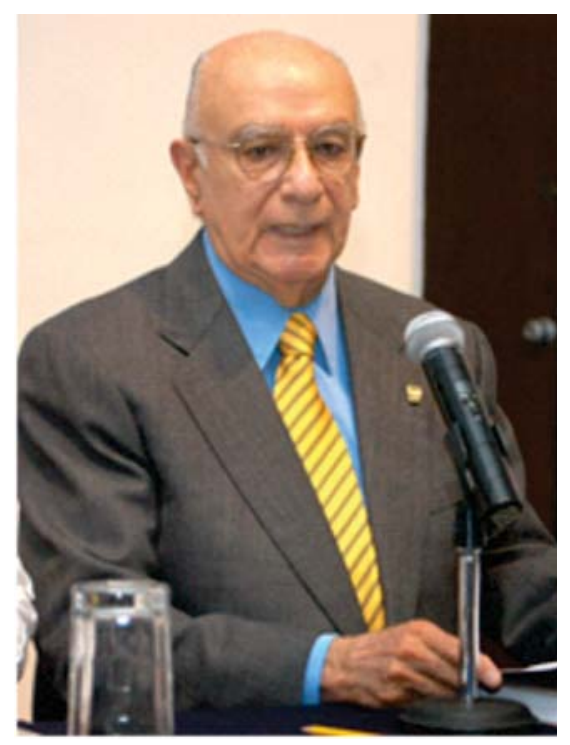

Dr. Octavio Rivero Serrano

Medalla Ismael Cosío Villegas-Donato G. Alarcón 2017

\section{Por: Francisco Navarro Reynoso}

El Dr. Octavio Rivero Serrano nació en la ciudad de Puebla el 15 de junio de 1929; sus padres fueron Gustavo Rivero y Teresa Serrano. Estudió la primaria, secundaria y preparatoria en la misma ciudad y terminó estos estudios en el año de 1946. Un año más tarde, ingresó a la Escuela Nacional de Medicina, fue practicante numerario del Hospital General de México y, habiendo terminado la carrera en 1952 ,

Este artículo puede ser consultado en versión completa en http://www.medigraphic.com/neumologia 
presentó el examen profesional los días 7 y 8 de mayo de 1953 en el cual obtuvo Mención Honorífica por la tesis titulada: Reconstrucción experimental de la tráquea. Fue médico aspirante del Hospital General de 1953 a 1954, donde presentó y ganó la oposición para médico adjunto en la Unidad de Neumología del mismo hospital; llegó a ser Jefe de la Unidad de Neumología durante el período de 1972 a 1977. También desempeñó el cargo de Subdirector Médico en esa dependencia. Sus principales cargos y reconocimientos son: Presidente de la Sociedad Mexicana de Neumología y Cirugía de Tórax (19651966), Director de la Facultad de Medicina en la Universidad Nacional Autónoma de México (1977-1981), Rector de la Máxima Casa de Estudios, la Universidad Nacional Autónoma de México (1981-1985), Embajador Extraordinario y Plenipotenciario de México en Roma, Italia (1986-1988), Coordinador del Programa Universitario de Medio Ambiente, UNAM, 1990-1994, Secretario del Consejo de Salubridad General, SSA. 1995-2001, Director del Seminario «El ejercicio actual de la medicina» dedicado a estudiar los cambios mostrados en el ejercicio de la medicina en los últimos años en la Facultad de Medicina de la UNAM (1997-2008), Presidente de la Academia Nacional de Medicina (1977), Reconocimiento como Profesor Emérito de la Facultad de Medicina por parte de la UNAM, Reconocimiento al Mérito Universitario a profesores e investigadores que cumplieron 50 años de labor académica, en la Ceremonia del Día del Maestro en la UNAM; Miembro Honorario de la Academia Nacional de Medicina y Miembro Honorario de la Academia Mexicana de Cirugía, Premio al Mérito en Salud del Consejo de Salubridad General en el marco del Día Mundial de la Salud: Condecoración «Doctor Eduardo Liceaga», abril 7, 2010, de manos del Presidente Lic. Felipe Calderón Hinojosa; Reconocimiento al Mérito Médico Institucional de la Sociedad Médica del Hospital General de México «Dr. Eduardo Liceaga» octubre, 2015, de manos del Presidente Lic. Enrique Peña Nieto.

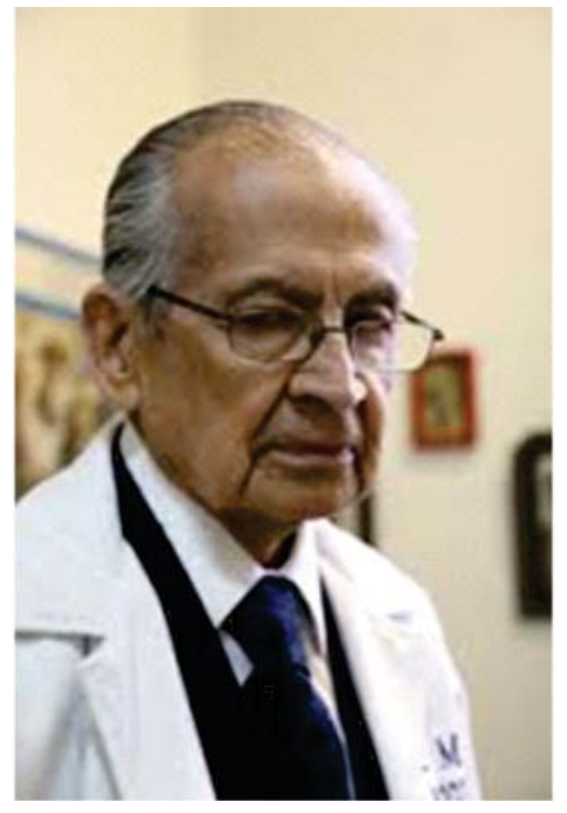

Dr. Raúl Cicero Sabido

Medalla Ismael Cosío Villegas-Donato G. Alarcón 2017

\section{Por: Alfredo Pérez Romo}

El Dr. Raúl Cicero nació en la sede de México en El Salvador, debido a las actividades diplomáticas de su padre; en sus años de adolescencia, además de estudios cortos de diplomacia, su inquietud y entusiasmo lo acercaron a una actividad deportiva que le daría sus primeros grandes logros, como el campeonato nacional de florete, ser miembro del equipo olímpico mexicano y de formar parte del Salón de la Fama Universitario de la UNAM, pero lo más importante fue su decisión de ingresar a la Escuela de Medicina, donde guiado por otro médico enorme de la medicina mexicana, el Dr. Alejandro Celis, y a través de su habilidad en la práctica médico quirúrgica, se incorporó al Hospital General de México como profesor ayudante de la Clínica del Aparato Respiratorio. En los años 50 fue el primer médico en el mundo en opacificar los linfáticos del aparato respiratorio, y junto con otros miembros del grupo del Dr. Alejandro Celis siguieron sus investigaciones en al campo vascular del pulmón. En 1980, por concurso fue nombrado profesor titular «C» de la Facultad de Medicina de la UNAM en el área de neumología y cirugía de tórax. Es profesor titular del Curso de Endoscopia Torácica, además de ser miembro del Comité Académico de Neumología en Posgrado de la UNAM. Ha dirigido 39 tesis de licenciatura, 35 de especialidad y cinco de maestría. En el ámbito de la investigación, el doctor Cicero es investigador nivel II del Sistema Nacional de Investigadores del CONACYT desde 1986; es también investigador en ciencias médicas categoría «F» por parte de la Coordinación General de Institutos Nacionales de Salud desde el 2006 y se ha destacado en las líneas de investigación de tuberculosis, cirugía de tórax y pleura y endoscopia torácica. A la fecha, numerosos trabajos suyos han sido publicados en revistas médicas internacionales, contando con más 250 publicaciones, entre las cuales destacan: 18 capítulos de libro, dos manuales (incluyendo el manual operativo de la CCT «Papel del médico general en el abandono del hábito de fumar») y 37 artículos de tuberculosis pulmonar. El doctor Cicero ha participado en la elaboración de programas contra el tabaquismo, tuberculosis y el Programa Universitario de Especialidades Médicas. Debido a su vasta trayectoria, tanto en la clínica como en el campo de la investigación, ha recibido diversas distinciones. En el año 2000 recibió de parte de la Secretaría de Salud el Premio a la Excelencia Médica y el Premio Nacional de Investigación Clínica «Miguel Otero». También ha sido merecedor de reconocimientos como el de la Academia Mexicana de Cirugía en 1993, el del Consejo Nacional de Cirugía de Tórax en el 2000, el «Reconocimiento por Lucha Anti-Tuberculosis» de la Secretaría de Salud en el 2001, el reconocimiento como «Cirujano Emérito» del Instituto Nacional de Ciencias Médicas en el año 2003 y el reconocimiento «Neumólogo formador de neumólogos» por la Sociedad de Neumología y Clínica de Tórax de México en el 2005. El doctor Cicero recibió la medalla «Mérito Universitario» de la UNAM en el 2004. El 31 de mayo de 2012 fue reconocido por la Secretaría de Salud por impulsar la lucha contra el tabaquismo en nuestro país, y ser pionero en la apertura de programas de cesación en la adicción de tabaco. Además de su amplia labor en la Secretaría de Salud 
y la Universidad Nacional Autónoma de México, el doctor Cicero es también miembro de la Academia Nacional de Medicina, la Academia Mexicana de Ciencias, la Academia Mexicana de Cirugía, The American Thoracic Society así como Fellowship del ACCP, entre otras. El Dr. Raúl Cicero Sabido fue Presidente de la SMNyCT en el bienio 1966-1967. Un mérito a desatacar es que es la única persona que ha asistido a todos los congresos celebrados por la SMNyCT desde 1944 hasta la fecha actual.

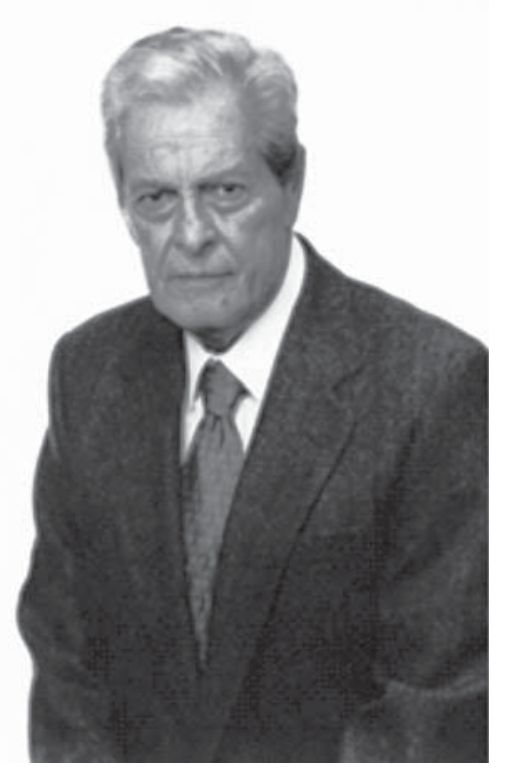

Dr. Jaime Villalba Caloca

Medalla Ismael Cosío Villegas-Donato G. Alarcón 2017

Por: Jorge Salas Hernández

El Dr. Jaime Villalba es médico cirujano egresado de la Facultad de Medicina de la Universidad de San Luis Potosí. En 1956 ingresó al Sanatorio para Enfermos de Tuberculosis de Huipulco, dirigido en ese momento por el Dr. Ismael Cosío Villegas y bajo la tutela directa del Dr. Fernando Rébora Gutiérrez. Poco tiempo después fue becado para complementar su proceso de formación en neumología, broncoscopia y cirugía de tórax en el Instituto Carlo
Forlanini, en la ciudad de Roma, Italia. El Dr. Villalba ha sido un incansable estudioso, prueba de esto es que al término de su gestión como Director General del INER, realizó el Doctorado en Ciencias, obteniendo el grado en el año 2008. Además, ha sido Profesor de Pregrado de la Clínica del Aparato Respiratorio y tutor de pasantes de medicina; en posgrado, profesor titular de la subespecialidad de cirugía toracopulmonar, Profesor Titular del curso de especialización en Neumología, miembro del Comité Académico en el área de Cirugía Cardiovascular y Torácica y Coordinador del Comité Académico en el área de Neumología del Programa Único de Especializaciones Médicas. Tutor de diversos programas de pregrado y de investigación. Ha participado en la dirección y coasesoría en tesis de licenciatura, especialidad, maestría y doctorado. También ha sido conferencista en los más importantes eventos académicos de la especialidad en Iberoamérica. En relación con su labor científica, ésta se ha enfocado a la investigación en trasplante pulmonar, trasplante de tráquea, cáncer pulmonar, tuberculosis, tabaquismo y contaminación ambiental. Todos recordamos su liderazgo en el grupo multidisciplinario que realizó el primer trasplante pulmonar en México y América Latina en 1989. Tiene más de 100 publicaciones en revistas nacionales e internacionales, 17 capítulos de libro, 19 editoriales y más de 200 trabajos presentados en congresos nacionales e internacionales. Actualmente es miembro activo en la Academia Nacional de Medicina, Academia Mexicana de Cirugía, Sociedad Mexicana de Neumología y Cirugía de Tórax, de la que fue Presidente (1989-1991), Consejo Nacional de Neumología, Consejo Nacional de Cirugía del Tórax, American Thoracic Society, European Respiratory Society, Asociación Latinoamericana de Tórax, Sociedad Mexicana de Cirujanos Torácicos Generales, Asociación Mexicana de Investigadores de los Institutos Nacionales de Salud y Hospitales de Alta Especialidad y de la Red de Biomateriales e Ingeniería de Órganos y Tejidos. También continúa laborando intensamente en el INER como investigador en ciencias médicas E por la Comisión Externa de Investigación de los Institutos Nacionales de Salud y es Investigador Nacional Nivel 1 del Sistema Nacional de Investigadores. Es Jefe de la Unidad de Trasplante Pulmonar Experimental y del Departamento de Investigación en Cirugía Experimental, así como Presidente del Comité Hospitalario de Bioética y Vocal en el Comité Interno de Trasplante Pulmonar del INER. Por estas y muchas actividades más ha recibido múltiples premios y distinciones como el Premio Nacional de Administración Pública, el Premio Nacional de Cirugía «Dr. Francisco Montes de Oca» de la Academia Mexicana de Cirugía, Premio a la Excelencia Médica por ser pionero en el campo de trasplantes de órganos y tejidos en nuestro país en el Siglo XX, entregado por el Presidente de la República, durante la Ceremonia Conmemorativa del Día del Médico en el año 2000, Director General del Instituto Nacional de Enfermedades Respiratorias en el período 1993-2003, Consultor Honorífico de la Campaña Nacional contra la Tuberculosis, reconocimiento por 55 años de servicio en el Instituto Nacional de Enfermedades Respiratorias Ismael Cosío Villegas y reconocimiento al Mérito Universitario por 50 años de servicio en la Universidad Nacional Autónoma de México. Recién, la Academia Mexicana de Cirugía le otorgó el homenaje «Académico Doctor Clemente Robles Castillo», año 2016, como un reconocimiento a su excepcional dedicación a la práctica, enseñanza e investigación de la cirugía.

\section{$\triangle$ Correspondencia:}

Dr. Juan Carlos Vázquez-García Dirección de Enseñanza, Instituto Nacional de Enfermedades Respiratorias Ismael Cosío Villegas.

Calzada de Tlalpan Núm. 4502, colonia Sección XVI, 14080,

Del. Tlalpan, Ciudad de México.

Teléfono: 555487 1743, ext. 5146

Correo electrónico: drjcvazquez@gmail.com

El autor declara no tener conflicto de intereses. 